\title{
Wireless Tear Glucose Sensor System
}

\author{
A. Hennig ${ }^{1}$, J. Lauko' ${ }^{2}$ A. Grabmaier ${ }^{1}$, C. Wilson ${ }^{2}$ \\ ${ }^{1}$ Fraunhofer IMS, Finkenstrasse 61, 47057 Duisburg, Germany \\ ${ }^{2}$ NovioSense B.V., Industrieterrein Winkelsteeg, Transistorweg 5, 6534 AT Nijmegen, the Netherlands \\ andreas.hennig@ims.fraunhofer.de
}

\begin{abstract}
:
A novel wireless tear glucose level sensor system for diabetes patients is presented. The concept provides a miniaturized sensor that can be worn non-invasively under the eye lid. It is composed of a chronoamperometric glucose sensor and an ASIC set with integrated potentiostat and transponder circuits. Wireless energy and data transmission according to the passive transponder standard ISO18000-3 is used to power and readout the sensor by a reader unit. The special coil shape design of the sensor unit enables adoption to the shape of an eye whilst and hence high comfort for the patient. High integration level is achieved by a combination of antenna and sensor electrode wires.
\end{abstract}

Key words: Wireless, Glucose Sensor, Amperometric, Diabetes, Tear Glucose Level

\section{Introduction}

Diabetes is a widespread disease with approximately 285 million cases worldwide as of 2013 [1]. This work focuses on the continuous monitoring of glucose levels for type 1 and severe type 2 diabetes mellitus. In the case of diabetes type 1 an autoimmune destruction of insulin producing beta cells in the pancreases leads to a rapid decline in the availability of insulin and results in increased levels of glucose in blood and urine. In the case of type 2 diabetes insulin is produced by beta cells however the body gradually builds a resistance to the normal level of insulin known as insulin resistance. The consequences of diabetes that present a high burden on global health care systems are secondary diseases such as vascular disease resulting in higher occurrence of heart attack or stroke or the eventual loss of limbs and the formation of chronic wounds in diabetic foot ulcers and impairment of vision, renal failure as a result of diabetic retinopathy. To prevent the onset of this secondary disease, a degree of control and continual monitoring and response to blood glucose level is essential. Therefore the patent has to determine not only blood glucose levels at set points during the day but the patient should also be alerted to the onset of sudden changes in the levels of glucose. The majority of Type 1 and severe Type 2 diabetics control glucose levels by needle pricks and subsequent blood glucose strip testing. It is widely accepted but has several disadvantages to the patent. Needle pricks are painful, especially in infants and young children, as well as gradual loss of feeling in the extremities of the fingers as the consequence of continual finger prick testing. In addition to the pain and inconvenience of taking a finger prick the blood measurement offers only a single snapshot in time, insufficient to warn of rapid onset of hypoglycemic events that in the extreme can trigger loss of consciousness and even death. For the reasons mentioned researchers are looking for an alternative solution, many methods exist for the continual monitoring of glucose levels via a minimally invasive sensor placed through the skin [2]. However these methods although promising for the measurement of glucose trends also have additional draw backs such as infection and gradual loss of sensitivity. A promising approach is to take advantage of the correlation of glucose levels in tear fluid against that of blood glucose levels [3][4]. In this paper we describe a system for monitoring of the glucose levels in the basal tear fluid present in the eye.

Extensive research has been carried out on the correlation of tear and blood glucose levels since the first reports of van Harringen Survey of ophthalmology, 1981 and March Diabetes Care, 1982. Although the glucose levels in the eye were shown to correlate with the blood glucose levels, a delay of 15-20 minutes has been observed, as illustrated in Fig. 1. 


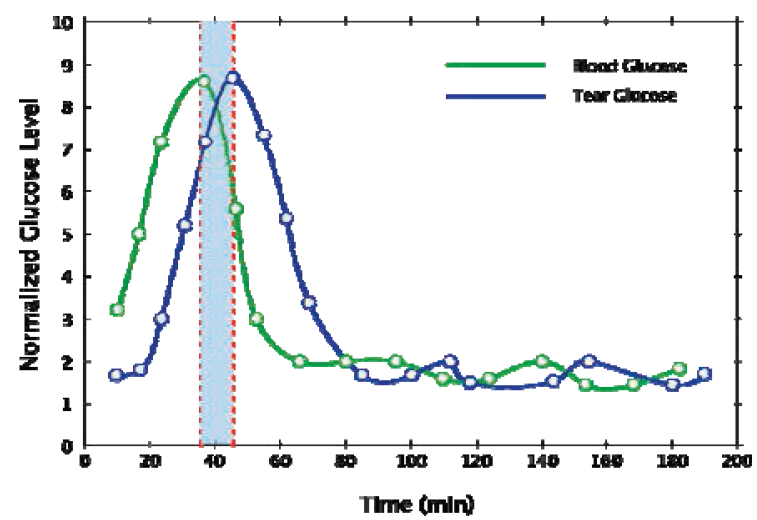

Fig. 1: Illustration of correlation between blood and tear glucose level

Furthermore the glucose concentrations in the tear fluid were generally found to be an order of magnitude lower than those in blood, ranging from 0.1 to $0.6 \mathrm{mmol} / \mathrm{L}$ [van Harringen, Survey of ophthalmology, 1981; Lane, Curr. Eye Research 2006; Foulks, Clinical Science 2007; Mitsubayashi, Talanta 2012). Some studies have also shown, that the levels were dependent on the sampling technique an excellent review on the subject is presented by Asher et al covering measurements reported in the period 1930 to 2007 [Asher, Clinical Science 2007]. These findings opened the possibility for a non-invasive glucose sensor, which could be placed into the eye. The reports of Baca et al along with that of Marcha and Lane indicated that tears are not all equal in concentration of glucose and therefore a method to directly measure "normal" or basal tear production is essential.

\section{Concept}

Based on the knowledge that basal tear production has to be directly measured a method was developed to measure glucose level directly under the bottom eyelid. In order to develop a device that not only fits into the lower eye lid but also has the capacity to conform to the surface of the eye and to eliminate the requirement to have any wires in or out of the lower eyelid a coil shaped device was chosen. A coil represents a device of high flexibility perpendicular to the short axis that is able to adopts to the shape of an eye whilst presenting an ideal device to act as an antenna for wireless data and power. To be able to measure glucose levels amperometricaly, two or three electrodes are coiled in parallel to form an amperometric cell while a forth wire is used to facilitate wireless data and power.

Fig. 2 illustrates the wireless tear glucose sensor system. It consists of the reader unit and the sensor unit. The reader unit generates a magnetic field in order to provide the sensor unit with energy. It receives and processes the measurement data captured by the sensor unit. Further it has a user interface to display the measurement result. The sensor unit is composed of a transponder, a potentiostat circuit and a glucose sensor. The transponder circuit handles the communication to the reader unit and is responsible for the energy management. The potentiostat circuit performes the amperometric measurement.

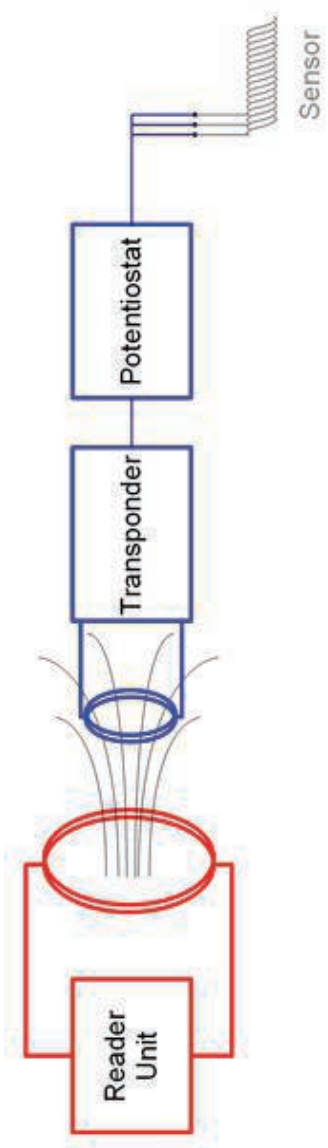

Fig. 2: System Concept

The glucose sensor uses a well-established and robust enzymatic detection of glucose. The enzyme glucose oxidase is a homodimeric glycoprotein, with a flavin adenine dinucleotide (FAD) cofactor. FAD plays an important role in the oxidation of glucose to gluconolactone, and in the course of the reaction, FAD is reduced to $\mathrm{FADH}_{2}$. The $\mathrm{FADH}_{2}$ is subsequently oxidized back to FAD by molecular oxygen, producing $\mathrm{H}_{2} \mathrm{O}_{2}$. The $\mathrm{H}_{2} \mathrm{O}_{2}$ produced is detected on a working electrode using chronoamperometrical measurement. This is a technique, where the current is measured as a function of time, directly after the introduction of a change in potential. 


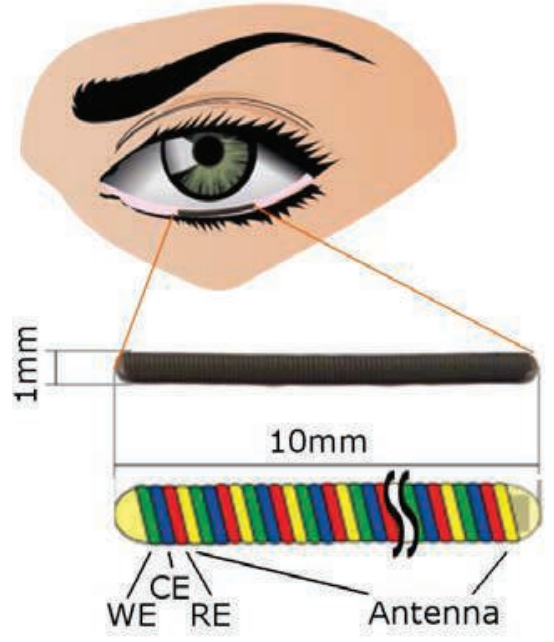

Fig. 3: Sensor Unit Concept

In order to achieve a highly integrated level of the sensor unit the concept provides an integration of the antenna coil and the sensor electrodes in form of a coil which enables high flexibility and comfort for the patient. Fig. 3 shows the configuration of electrode wires and antenna wire in the sensor unit. The antenna wire is colored yellow. The WE electrode is green. The CE electrode is blue and the RE electrode is reed. As can be seen the three electrode wires as well as the antenna wire are wound together to form the coil. Over a length of $10 \mathrm{~mm}$ approximately 15 turns can be incorporated. The ASIC is placed at one end of the coil. There the tree electrodes as well as the antenna wire are connected.

A passive transponder technology is well suited for glucose level monitoring where quasi continuous measurements with sample times in the order of a few minutes to a few hours are sufficient. Therefore a passive transponder coupled to the amperometric glucose sensor was used. This makes a wireless readout of the glucose sensor placed under the eye lid possible. Thus the battery-free operation enables miniaturization and allows the sensor to be placed unobtrusively under the lower eyelid. Basically passive transponder systems work in the LF (100 kHz to $133 \mathrm{kHz})$, HF (13.56 $\mathrm{MHz})$ or UHF (868 MHz) Bands. Each frequency has different physical properties. Especially antenna characteristics and damping effects of the human tissue differ at each frequency band. Suitable to the coil shape design of the antenna inductive coupling principle was chosen. To find out the best configuration of the reader coil as well as the best operating frequency a 3D field simulation was performed. The simulation software uses a finite integral method (FIM). Fig. 4 shows the generated model made of an simplified human eye, an eye lid, the coil of the sensor unit and the coil of the reader unit. The dielectric properties of human tissue were used that can be found in [5].

Theoretically the damping of human tissue will increase with the frequency. In contrast to the frequency depending damping effects, the induced voltage at the transponder coil increases with the frequency in accordance with the induction law. It was found out, that the HF band is well suited for these kinds of applications. At this frequency the damping of the tissue is rather low and the induction of voltage in the sensor unit coil is at an acceptable level. For operation of the circuit minimum voltage amplitude is required. The coil axis will be orientated in a direction along the eye lid. Therefore the reader coil has to generate a magnetic field with the same orientation at the location of the helix. Due to the limited length of the helix and the minimum practicable wire diameter the number of windings of the antenna coil is 15 turns. This leads to the fact that the antenna coil cannot be dimensioned to achieve optimal matching to the transponder circuit load impedance. Instead, the transponder load impedance has to be tuned to the antenna coil parameters.

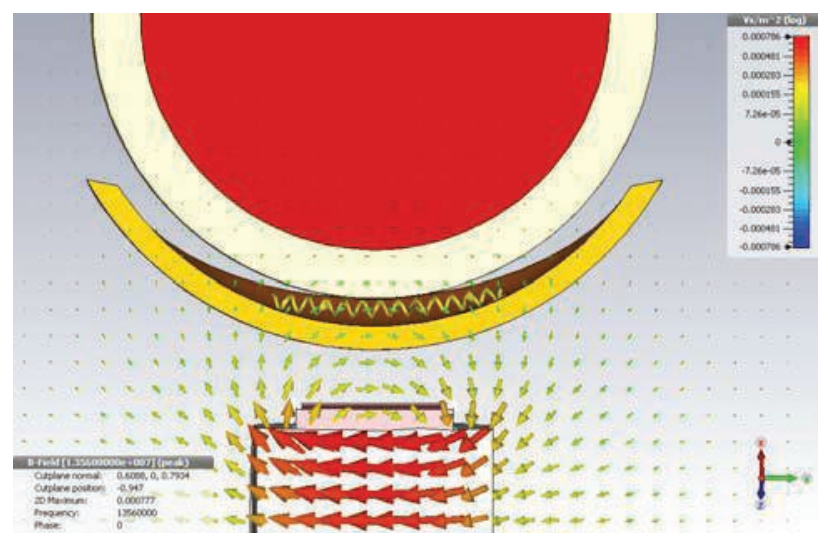

Fig. 4: 3D field simulation of inductive link

The task of the transponder circuit is to generate a stable supply voltage for the potentiostat and handle the communication between the reader unit and the potentiostat citcuit. For this purpose it has a rectifier connected to the antenna coil. Between the transponder and the potentiostat there is a digital SPI interface. Hence the transponder system acts as a bridge between the reader and the potentiostat. With the help of the SPI interface the potentiostat can be controlled and digitalized sensor currents can be read out.

\section{Realization}

The ASIC set shown in Fig. 5 was developed that performs the chronoamperometric 
measurement and to generate a stable supply voltage for the potentiostat circuit as well as handle the communication between the reader unit and the potentiostat circuit. The ASICs were fabricated in $350 \mathrm{~nm}$ CMOS technology.

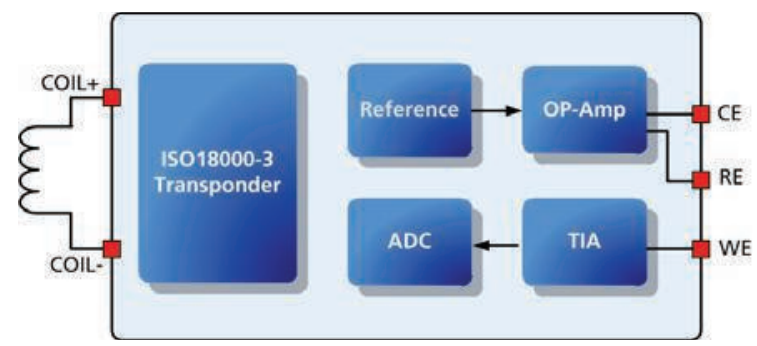

Fig. 5: Funktional block diagramm of ASIC set

The fabricated ASICs and the fabricated helix were assembled to a sensor unit. Fig. 6 shows a first prototype of the assembled sensor unit.

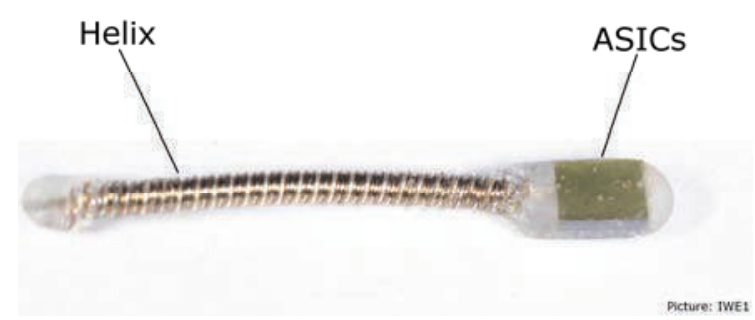

Fig. 6: first prototype of sensor unit

The ASICs are bonded on a flexible PI film using flip chip technique. The helix wires are connected to the $\mathrm{PI}$ film using conductive adhesive. An encapsulation protects the ASICs against liquids.

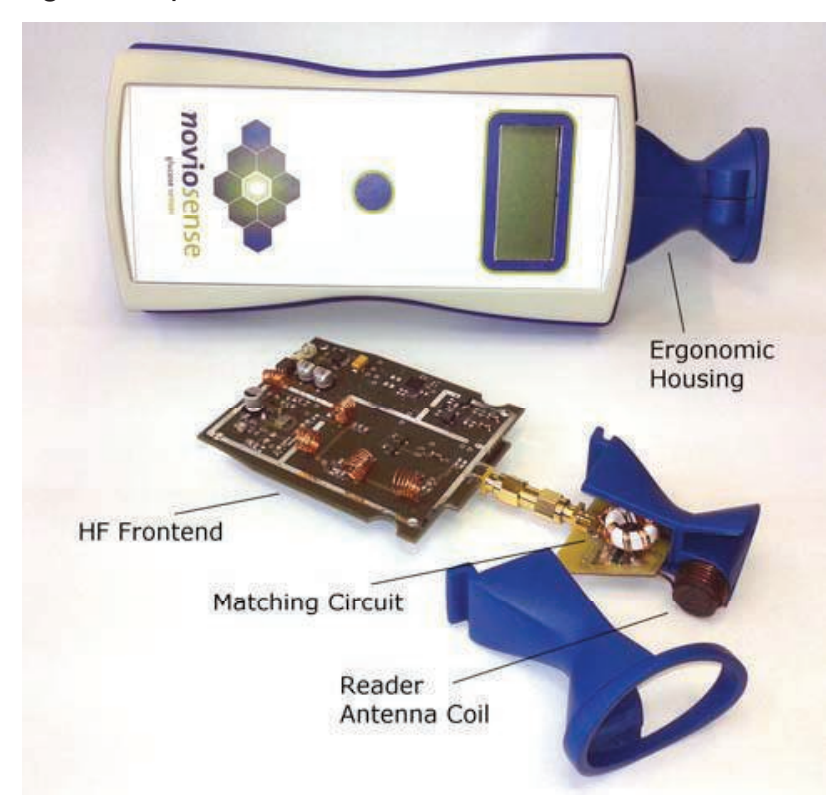

Fig. 7: Prototype of reader unit with NFC frontend

Fig. 7 shows the developed reader unit. A special antenna housing was designed that has an ergonomically form for the use at the patient eye. It is placed in the top of the reader case. The reader unit has a simple user interface consisting of a LCD display and one button. One-button operation makes the device easy to use. The user places the ergonomic housing near his eye in such a way, that the reader coil is in the proximity of the sensor unit. After pressing the button, the HF frontend is activated and the magnetic RF field is generated. The sensor unit is powered by the field. After that, the reader reads out the ID number of the sensor unit and downloads calibration data of the sensor if this is not already done in a previous readout. Then a measurement is initiated. After the corresponding measurement time the result is read out. The reader unit calculates the real glucose level using calibration data and measurement values. The determined glucose level is displayed and can be read by the user. The reader is powered by a rechargeable lithium battery. A $0.8 \mathrm{~mm}$ copper wire wound around a ferrite rod is used as a reader coil. The reader coil is placed inside the ergonomically housing together with a matching circuit. The position of the antenna coil in the housing is chosen in a way that the coil is near the eye lid.

\section{Results}

To analyze the performance and linearity of the glucose sensor a measurement with reference devices is done. Thereby a dummy cell composed of a resistor network with known impedances is used. Fig. 8 shows the resulting ADC counter values over the calculated currents in the dummy cell. A good linearity can be seen in the target measurement range.

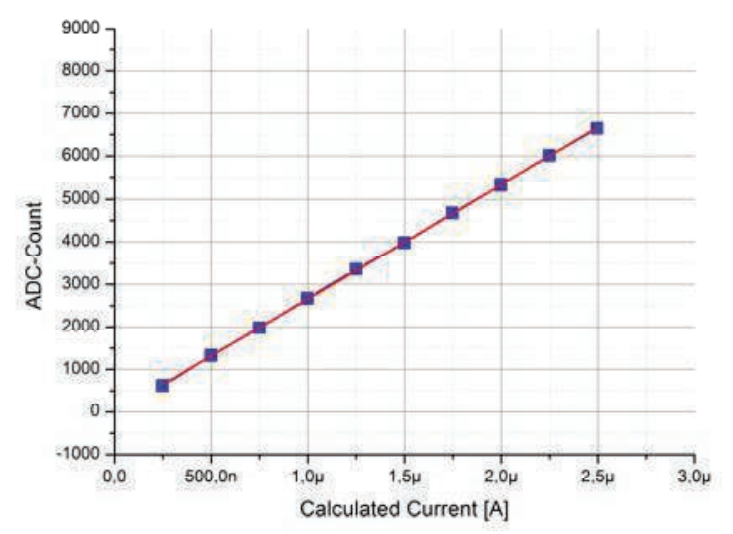

Fig. 8: Linearity of the potentiostat

To demonstrate the working system, six sample tubes are prepared. In each a buffer solution with different glucose concentrations from 0.1 $\mathrm{mMol}$ to $1 \mathrm{mMol}$ are filled in. The glucose 
sensor was dipped into each sample tube and a measurement was performed. The WE electrode current measured by the potentiostat is plotted in Fig. 9.

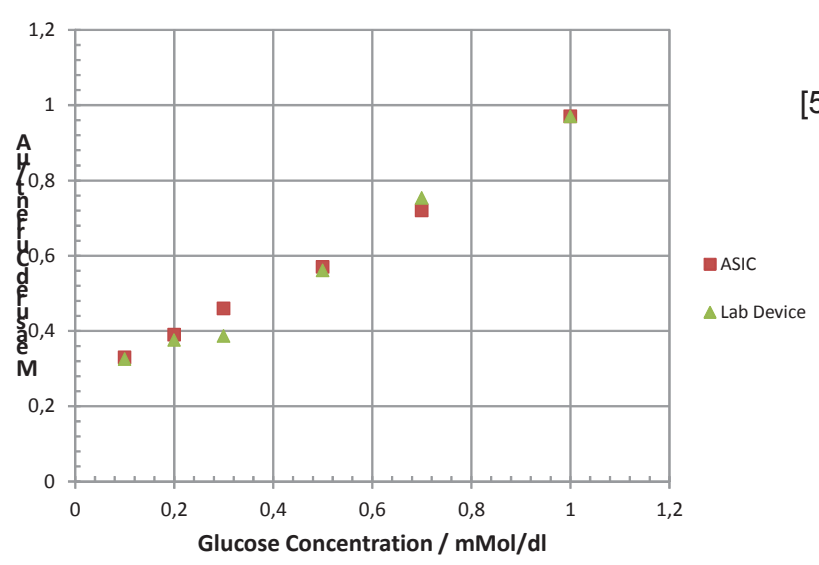

Fig. 9: Measurement results

For reference purposes a commercial lab potentiostat (from Autolab) was used to verify the measured currents. Each point of the figure represents a WE electrode current sampled 15 seconds after applying the potential to the CE electrode. As can be seen, the sensor unit provides acceptable results.

\section{Conclusion}

In this paper a novel wireless tear glucose monitoring system was presented. The sensor concept is based on chronoamperometric measurement. Wireless energy and data transmission was realized using inductive coupling at an operating frequency of 13.56 $\mathrm{MHz}$. A novel design of integrated antenna and electrode wires enables high degree of miniaturization. A coil shape design of the sensor unit enables adoption to the shape of an eye whilst. A complete demonstrator system was developed including ASIC fabrication, assembly technique and reader unit manufacturing. Finally the functionally was demonstrated in a laboratory environment.

The results were promising. After a re-design phase the system will be tested under clinically relevant conditions.

\section{References}

[1] Dixon JB; Zimmet P; Alberti KG; Rubino F; Bariatric surgery: an IDF statement for obese Type 2 diabetes; International Diabetes Federation Taskforce on Epidemiology and Prevention

[2] Sandeep Kumar Vashist; Continuous Glucose Monitoring Systems: A Review; Diagnostics 2013, 3, 385-412; doi:10.3390/diagnostics3040385
[3] N. J. Van Haeringen, Survey of Ophthalmology 1981, 26, 84-96; b) J. D. Lane, D. M. Krumholz, R. A. Sack and C.

[4] Chu M et al 2011 Biomedical soft contact-lens sensor for in situ ocular biomonitoring of tear contents Biomed. Microdevices

[5] S Gabriel, R W Lau, and C Gabriel. The dielectric properties of biological tissue: lii. Parametric models for the dielectric spectrum of tissues. Phys. Med. Biol. 41, 1996. 